\title{
Plasmon-enhanced terahertz emission in self-assembled quantum dots by femtosecond pulses
}

\author{
F. Carreño, ${ }^{1, a)}$ M. A. Antón, ${ }^{1, b)}$ Sonia Melle, ${ }^{1, c)}$ Oscar G. Calderón, ${ }^{1, d)}$ \\ E. Cabrera-Granado, ${ }^{1, e)}$ Joel Cox, ${ }^{2, f)}$ Mahi R. Singh, ${ }^{2, g)}$ and A. Egatz-Gómez ${ }^{3, h)}$ \\ ${ }^{1}$ Facultad de Óptica y Optometría, Universidad Complutense de Madrid, C/ Arcos de Jalón 118, \\ 28037 Madrid, Spain \\ ${ }^{2}$ Department of Physics and Astronomy, The University of Western Ontario, London N6A 3K7, Canada \\ ${ }^{3}$ Department of Chemical and Biomolecular Engineering, University of Notre Dame, South Bend, \\ Indiana 46556, USA
}

(Received 31 October 2013; accepted 18 January 2014; published online 12 February 2014)

\begin{abstract}
A scheme for terahertz $(\mathrm{THz})$ generation from intraband transition in a self-assembled quantum dot (QD) molecule coupled to a metallic nanoparticle (MNP) is analyzed. The QD structure is described as a three-level atom-like system using the density matrix formalism. The MNP with spherical geometry is considered in the quasistatic approximation. A femtosecond laser pulse creates a coherent superposition of two subbands in the quantum dots and produces localized surface plasmons in the nanoparticle which act back upon the QD molecule via dipole-dipole interaction. As a result, coherent $\mathrm{THz}$ radiation with a frequency corresponding to the interlevel spacing can be obtained, which is strongly modified by the presence of the MNP. The peak value of the terahertz signal is analyzed as a function of nanoparticle's size, the MNP to QD distance, and the area of the applied laser field. In addition, we theoretically demonstrate that the terahertz pulse generation can be effectively controlled by making use of a train of femtosecond laser pulses. We show that by a proper choice of the parameters characterizing the pulse train a huge enhancement of the terahertz signal is obtained. (C) 2014 AIP Publishing LLC. [http://dx.doi.org/10.1063/1.4863781]
\end{abstract}

\section{INTRODUCTION}

Terahertz $(\mathrm{THz})$ technology is an ever growing field, due to the important properties of such radiation and its significant applications, in spectroscopy and imaging. The $\mathrm{THz}$ wave can be obtained by both electronic ${ }^{1}$ and optical methods. $^{2,3}$ Because of the lack of high quality terahertz sources and efficient detectors, the study and application of terahertz pulses have been greatly restricted. Recently, with the advent of the femtosecond laser technique, the reliable terahertz pulses can be obtained by photo-conductive switches, ${ }^{4,5}$ optical rectification $(\mathrm{OR})^{6}$ or photo-induced plasma in a gaseous medium. ${ }^{7,8}$ The development of quantum cascade laser $^{9-13}$ based on semiconductor super lattices is also a breakthrough for $\mathrm{THz}$ wave generation. Terahertz fields have been widely applied in various areas of research, such as non-invasive imaging, ${ }^{14,15}$ and have been described in several comprehensive review articles. ${ }^{16,17}$

One promising direction towards the realization of both detectors and emitters operating in the $\mathrm{THz}$, are semiconductor nanostructures, such as quantum wells (QWs) and quantum dots (QDs). ${ }^{18,19}$ Low-dimensional semiconductor heterostructures are almost ideally suited for terahertz and infrared generation because the spacing between levels of dimensional

\footnotetext{
a)ferpo@fis.ucm.es

b)antonm@fis.ucm.es

c) smelle@fis.ucm.es

d) oscargc@fis.ucm.es

e)ecabrera@fis.ucm.es

f) jcox27@uwo.ca

g) msingh@uwo.ca

h)Ana.Egatz-Gomez.1@nd.edu
}

quantization can be conveniently manipulated over the region from several to hundreds of microns, and injection pumping is possible. The specific working frequencies of such devices are determined by the structure design and their modification for a given fabricated sample is possible just by applying an external electric or magnetic field and by means of a mechanical stress.

Optically pumped terahertz emission from a semiconductor surface has been observed in a wide range of semiconductors. Several mechanisms for coherent $\mathrm{THz}$ emission from semiconductor heterostructure devices have been demonstrated in the past. In particular, QWs have been proven as $\mathrm{THz}$ emitters wherein $\mathrm{THz}$ generation is due to charge oscillations with wave packets tunneling back and forth between wells. For example, Roskos et al. ${ }^{20,21}$ and Planken et al. ${ }^{22}$ used near-infrared femtosecond laser pulses to excite quantum beats in undoped quantum wells. Generation of terahertz radiation based on the difference of optical frequencies in bound-bound transitions is one of the most promising mechanisms to produce coherent $\mathrm{THz}$ sources. ${ }^{23}$

In strong laser fields, the emission spectra contain a Rabi frequency that is much smaller than the frequency of the driven laser (located in the low frequency region). In this regime, investigations on few-cycle laser pulses interaction with asymmetric systems have been carried out along recent years. One should break the inversion symmetry of the atomic and molecular systems to obtain low frequency radiation corresponding to slow oscillations which can be realized by the degenerated energy levels combined with the initial coherent superposition states of a certain parity or applying a weak static electric field. The generation of terahertz transients from semiconductor irradiated by femtosecond laser 
pulses has been well-studied in the context of searching for intense, pulsed terahertz emitters. ${ }^{24-26}$ From the nonlinear optics point of view, the terahertz generation from bulk materials and quantum wells modeled as a three level system can be explained from the second-order nonlinear susceptibility for difference-frequency generation, $\chi^{(2)}\left(\omega_{1}-\omega_{2} ; \omega_{1}\right.$, $\left.\omega_{2}\right)$. Wiener ${ }^{27}$ calculated the time-dependent polarization for this system by using first order perturbation theory. The polarization for this system is proportional to the product of the transition dipole moments of the three transitions. In the case of a semiconductor heterostructure with a symmetric potential, one of the interband matrix elements is zero, so terahertz emission is impossible to occur. Even if the symmetry of the potential is broken by an electric field of a few $\mathrm{kV} / \mathrm{cm}$ the matrix element remains very small. Therefore, $\mathrm{THz}$ emission due to quantum beats cannot be expected very high. This limitation can be addressed by designing new, nano-engineered materials whose resonances are tuned to a frequency region of interest, such as specially designed plasmonic structures. It is well-known that dipole-dipole interactions occur naturally in nanometer-scale hybrid heterostructures. Nanometer-scale metallic structures can dramatically modify the optical properties of various optically active objects of similar dimensions such as atoms, molecules, or semiconductor quantum dots. In the presence of an external laser field, the surface plasmon oscillation in metallic nanoparticles (MNPs) renormalizes the external field and enhances the electric field in the QDs. The strong modifications are conceptually well understood as a product of free electron oscillations in the metal that induce strong localized electric fields near the surface of nanostructured metals. This has motivated that the research field of plasmonics, while still expanding its applications in linear nano-optics, is quickly advancing towards nonlinear phenomena opening a route to numerous practical applications, such as surface enhanced Raman scattering (SERS), ${ }^{28,29}$ the control of radiation from single quantum emitters ${ }^{30}$ or generation of extreme ultraviolet pulses by nonlinear high harmonic generation. ${ }^{31}$ From a theoretical point of view, many interesting phenomena have been found in these coupled nanocrystals, such as nonlinear Fano effect, ${ }^{32-34}$ Förster energy transfer, ${ }^{35}$ and local field enhancement. ${ }^{36,37}$ Such exciton-plasmon coupling can lead to a dramatic change in the optical properties. This includes enhancement or suppression of their emission, ${ }^{37}$ shift and broadening of their excitonic transitions, ${ }^{37,38}$ enhancement of the Rabi flopping, ${ }^{39}$ and optical bistability. ${ }^{40}$ Significant attention has been focused on the emerging field of quantum plasmonics with the goal of making devices for quantum information processing ${ }^{41,42}$ as single photon transistors $^{43}$ or lasers. ${ }^{44}$ Controlling light-matter interaction at the nanoscale has become as one of the paramount goals of nanophotonics. ${ }^{45-47}$ Additionally, the possibility of reaching the quantum regime using plasmonic systems has also been addressed. ${ }^{48-50}$

Here, we will discuss the terahertz generation enhancement from intraband transition in self-assembled QD molecules near a MNP. The linear and nonlinear optical properties in hybrid systems based on metal nanoparticles have attracted the attention primarily in surface-enhanced
Raman scattering. ${ }^{51}$ More recently there are few works on the enhancement of second harmonic generation ( $\mathrm{SHG}$ ), either in semiconductor QDs or in molecules, produced by the resonant excitation of metal nanoparticles. For instance, enhanced optical SHG by a factor of 8 has been observed in hybrid polymer nanoassemblies based on coupled surface plasmon resonance of a gold nanoparticle array. The observed enhancement was attributed to coupling of surface plasmons between two adjacent gold nanoparticles. ${ }^{52}$ Second harmonic generation properties of fluorescent polymer-encapsulated gold nanoparticles has been demonstrated. ${ }^{53}$ Midinfrared generation from difference frequency in self-assembled quantum dots near metal nanoparticles with two-color interband excitations have been theoretically analyzed, ${ }^{54}$ and SHG signals produced by quantum dot and nanoparticle have been theoretically and experimentally addressed in QD-MNP system. ${ }^{55-57}$

In this paper, we will analyze the ultrafast optical dynamics of terahertz radiation in a hybrid resonant plasmonexciton system. A short laser pulse excite heavy holes (hh) excitons from two adjacent QDs and creates a coherent superposition of two quasi-degenerated adjacent levels with dipole coupling in the quantum dot molecule, ${ }^{58,59}$ which in turn implies the existence of charge oscillations in the system. The level of overlapping of hole wave-functions can be tuned with the application of an external gate voltage. As a result, coherent excitation of $\mathrm{THz}$ radiation with a frequency corresponding to the inter-level spacing can be observed. Specifically, we discuss the plasmon enhancement terahertz generation from intraband transition in quantum dots by femtosecond pulses. It is shown that by tuning the pump field near the resonance of the two coupled exciton states the generated terahertz radiation can be enhanced depending on the size and localization of the MNP. In addition, when the hybrid system is driven by a coherent multipulse sequence, we show that the generated terahertz radiation can be either enhanced or weakened, by controlling the relative time delay and phase difference between the optical pulses. The paper is organized as follows: Sec. II establishes the model, i.e., the Hamiltonian of the system and the time-evolution equations of the atomic operators assuming the rotating wave approximation. Section III deals with the numerical simulations and discusses the enhancement of the terahertz emission with pulse trains. Section IV summarizes the main conclusions.

\section{THEORETICAL MODEL}

We investigate quantum coherence and interference phenomena in a QD-MNP hybrid system. We consider a spherical MNP with radius $a$ coupled to a QD (see Fig. 1). The center-to-center distance between the QD and the MNP is denoted as $R$. We consider a QD molecule consisting in two QD embedded in the intrinsic region of a typical p-i field effect structure. A suitable level scheme is provided by a pair of vertically stacked self-assembled InGaAs QDs, separated by a thin GaAs tunnel barrier and embedded in a GaAs Schottky diode. ${ }^{59,60}$ By applying an external gate voltage, the number of surplus holes in the dot structure can be precisely controlled. By tuning appropriately a external gate voltage, we have a knob to change the overlapping of the 
(a)

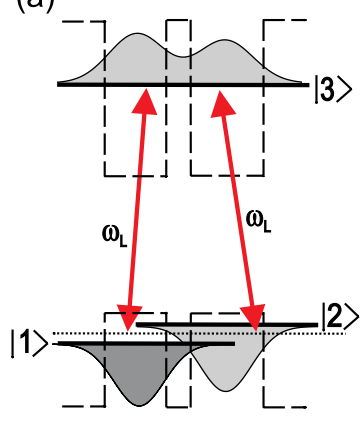

(b)

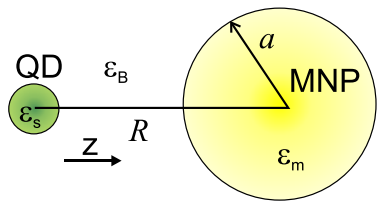

(c)

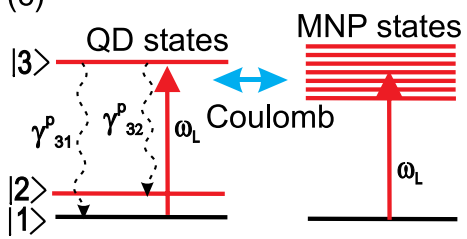

FIG. 1. (a) Schematic illustration of the proposed double-dot molecule. The dashed line indicates the confinement potential along the growth axis for electron (top) and holes (bottom) including an external electric field which allows for the control of overlapping of hole wave-functions. (b) The QDMNP hybrid system. The MNP has a radius $a$ and a dielectric constant $\epsilon_{m}$. The dielectric constants of the QD and the environment are $\epsilon_{s}$ and $\epsilon_{B}$, respectively. (c) Atomic states of the QD molecule and the MNP. $\gamma_{32}^{(0)} / \gamma_{32}^{p}$ and $\gamma_{31}^{(0)} / \gamma_{31}^{p}$ are the free space/plasmon-modified upper level decays to the ground levels, and $\omega_{L}$ stands for the angular frequency of the laser field.

hole wave-functions of the two dots. In addition, the electron wave function extends over the two dots because of the smaller electron mass and the resulting increased interdot tunneling. As a consequence, the full system can be represented by a three level $\Lambda$-type system. The three states are denoted as $|1\rangle,|2\rangle$, and $|3\rangle$, with energies $\hbar \omega_{j},(j=1-3)$. The two single-excitonic transitions $|1\rangle \leftrightarrow|3\rangle$ and $|2\rangle \leftrightarrow|3\rangle$ are orthogonal linearly polarized, ${ }^{61}$ or left and right circularly polarized. ${ }^{62}$ The resonant frequencies between the upper level $|3\rangle$, and the ground levels $|1\rangle$ and $|2\rangle$ are $\omega_{31}$ and $\omega_{32}$, respectively. Note that $\omega_{31}-\omega_{32}=\omega_{21}, \omega_{21}$ being the frequency separation of the ground levels. Levels $|1\rangle$ and $|2\rangle$ are also dipole coupled. It is to be noted that in semiconductor quantum wells or bulks modeled as three-level systems, all the transitions are not allowed because of parity.

A laser field $\vec{E}$ with frequency $\omega_{L}$ couples the ground levels $|1\rangle$ and $|2\rangle$ to the upper level $|3\rangle$, and is given by

$$
\vec{E}=\frac{1}{2}\left(E_{1}(t) \vec{u}_{1}+E_{2}(t) \vec{u}_{2}\right) e^{-i \omega_{L} t}+\text { c.c. },
$$

where $E_{1}\left(E_{2}\right)$ is the slowly varying amplitude along the $\vec{u}_{1}\left(\vec{u}_{2}\right)$ direction, which corresponds to the polarization of the transition $|1\rangle \leftrightarrow|3\rangle(|2\rangle \leftrightarrow|3\rangle)$.

We take into account that the effective size of the QD molecule is usually very small, thus, the QD structure can be considered as a point-like dipole induced by the external field. The radius $a$ of the MNP is on the nanometer scale and it is described in the framework of classical electrodynamics. The oscillating electric field of the driving laser will generate localized surface charge oscillations in the MNP and an electric dipole in the QD molecule, which interact with each other via the dipole-dipole interaction. The driving field is assumed to be spatially uniform for the relatively small dimension of the hybrid system, and the quasistatic approximation is used. The dielectric function of the QD is denoted as $\epsilon_{s}$, while that of the host medium is denoted as $\epsilon_{B}$ (see Fig. 1). Finally, the dielectric function of the MNP is $\epsilon_{m}(\omega)$.

The Hamiltonian of the hybrid system can be expressed as

$$
H=H_{0}+H_{E}+\mathcal{L} \rho,
$$

where

$$
\begin{aligned}
& H_{0}=\hbar \sum_{j=1}^{3} \omega_{j} \hat{\sigma}_{j j}, \\
& H_{E}=-\frac{1}{2} \sum_{j=1}^{2} \mu_{j 3} E_{Q D}^{(j)} e^{-i \omega_{L} t} \hat{\sigma}_{3 j}+H . c .
\end{aligned}
$$

where $\mathcal{L} \rho$ is the Liouvillian of the system and H.c. stands for the Hermitian conjugate. The parameters $\mu_{j 3}$ with $j=1,2$ are the interband dipole matrix elements of the excitonic transitions $|3\rangle \rightarrow|1\rangle$ and $|3\rangle \rightarrow|2\rangle$, respectively. $E_{Q D}^{(j)}(j=1,2)$ is the slowly varying amplitude of the total field felt by the QD polarized along the $|3\rangle \leftrightarrow|j\rangle$ transition, which is given by

$$
E_{Q D}^{(j)}=\frac{1}{\epsilon_{e f f s}}\left[E_{j}+\frac{1}{4 \pi \epsilon_{0} \epsilon_{B}} \frac{S_{j} P_{M N P}^{(j)}}{R^{3}}\right](j=1,2),
$$

where $\epsilon_{\text {effs }}=\left(2 \epsilon_{B}+\epsilon_{s}\right) / 3 \epsilon_{B}$, and $S_{j}$ is 2 or -1 when the electric field $E_{j}$ is polarized along the axis of the hybrid system or in the orthogonal direction. The $Z$ direction corresponds to the axis of the hybrid system (see Fig. 1). The dipole $P_{M N P}^{(j)}$ originates from the charge induced on the surface of the MNP, and depends on the total field due to the QD (Ref. 62) as

$$
P_{M N P}^{(j)}=4 \pi \epsilon_{0} \epsilon_{B} a^{3} \gamma\left(\omega_{L}\right) E_{M N P}^{(j)}(j=1,2),
$$

where $E_{M N P}^{(j)}$ is the slowly varying field amplitude at frequency $\omega_{L}$ felt by the MNP, which is given by

$$
E_{M N P}^{(j)}=E_{j}+\frac{1}{4 \pi \epsilon_{0} \epsilon_{B}} \frac{S_{j} P_{Q D}^{(j)}}{R^{3}}(j=1,2),
$$

and $\gamma\left(\omega_{L}\right)=\left[\epsilon_{m}\left(\omega_{L}\right)-\epsilon_{B}\right] /\left[2 \epsilon_{B}+\epsilon_{m}\left(\omega_{L}\right)\right]$ is the dipole polarizability of the MNP. The dipole $P_{Q D}^{(j)}(j=1,2)$ is expressed via the off-diagonal elements of the density matrix as follows:

$$
P_{Q D}^{(j)}=\mu_{j 3} \rho_{3 j} .
$$

Substituting Eqs. (6) and (7) back into Eq. (5), we obtain

$$
P_{M N P}^{(j)}=2 \pi \epsilon_{0} \epsilon_{B} a^{3} \gamma\left(\omega_{L}\right)\left(E_{j}+\frac{1}{2 \pi \epsilon_{0} \epsilon_{B}} \frac{S_{j} \mu_{j 3} \rho_{3 j}}{R^{3}}\right)(j=1,2) .
$$

Finally, the slowly varying amplitudes of the fields in the QD are

$$
E_{Q D}^{(j)}=\frac{E_{j}}{\epsilon_{\text {effs }}}\left(1+\frac{S_{j} a^{3} \gamma\left(\omega_{L}\right)}{R^{3}}\right)+\frac{S_{j}^{2} a^{3} \gamma\left(\omega_{L}\right) \mu_{j 3} \rho_{3 j}}{2 \pi \epsilon_{0} \epsilon_{B} \epsilon_{\text {effs }} R^{6}}(j=1,2) .
$$


By introducing Eq. (9) into Eq. (3), the total Hamiltonian of the QD in the dipole approximation can be expressed as follows:

$$
\begin{aligned}
& H_{0}=\hbar \sum_{j=1}^{3} \omega_{j} \hat{\sigma}_{j j}, \\
& H_{E}=-\hbar \sum_{j=1}^{2}\left(\Omega_{j}+G_{j} \rho_{3 j}\right) \hat{\sigma}_{3 j} e^{-i\left(\omega_{L}-\omega_{3 j}\right) t}+H . c .
\end{aligned}
$$

where we have introduced the magnitudes $\Omega_{j}$ and $G_{j}$, which contain the effects of both coupling fields and localized surface plasmons, and are explicitly given by

$$
\begin{aligned}
\Omega_{j}^{0} & =\frac{\mu_{j 3} E_{j}}{2 \hbar \epsilon_{e f f s}}, \\
\Omega_{j} & =\Omega_{j}^{0}\left[1+\frac{S_{j} a^{3} \gamma\left(\omega_{L}\right)}{R^{3}}\right](j=1,2), \\
G_{j} & =\frac{S_{j}^{2} \mu_{j 3}^{2} a^{3} \gamma\left(\omega_{L}\right)}{4 \pi \epsilon_{0} \epsilon_{B} \hbar \epsilon_{e f f s} R^{6}} .
\end{aligned}
$$

In the above equations, $\Omega_{j}$ is the renormalized Rabi frequency associated with the driving field and the field produced by the induced dipole moment $P_{M N P}$ of the MNP. In addition, $G_{j}$ shows the interaction between the polarized QD and the MNP. Note that $G_{j}$ is a complex quantity. When the laser field is weak, the imaginary part of $G_{j}$ represents the Förster energy transfer rate from the QD to the MNP and therefore contributes to the damping rate of the QD. The real part of $G_{j}$ refers to the red shift of the QD transition caused by the plasmonic effects. ${ }^{33,39}$

In view of the previous considerations, the time evolution of the density matrix now reads

$$
\frac{\partial \rho}{\partial t}=-\frac{i}{\hbar}[H, \rho]-\mathcal{L} \rho .
$$

The term $\mathcal{L} \rho$ which accounts for the spontaneous decay rates of the involved transitions, can be written in the Lindblad form

$$
\begin{aligned}
\mathcal{L} \rho= & \frac{\gamma_{31}^{p}}{2}\left(\rho \sigma_{33}+\sigma_{33} \rho-2 \sigma_{13} \rho \sigma_{31}\right) \\
& +\frac{\gamma_{32}^{p}}{2}\left(\rho \sigma_{33}+\sigma_{33} \rho-2 \sigma_{23} \rho \sigma_{32}\right) \\
& +\frac{\gamma_{21}}{2}\left(\rho \sigma_{22}+\sigma_{22} \rho-2 \sigma_{12} \rho \sigma_{21}\right) .
\end{aligned}
$$

Here, $\gamma_{3 j}^{p}(j=1,2)$ stand for the spontaneous emission decay rates of the QD. These parameters are modified by the plasmonic field of the MNP. The dissipative process described by the term of the Liouvillian with the pre-factor $\gamma_{21}$ accounts for the lower levels' dephasing and is assumed to be uncoupled with the localized surface plasmons due to the low values of the Zeeman splitting considered.

We consider that the QD molecule can lose its excitation radiatively by emitting a photon and non-radiatively through the dissipation of currents induced by the QD in the MNP. In order to describe how the spontaneous emissions of the QD's atomic transitions are modified by the plasmonic interaction, we make use of the classical calculations of the fields as shown, for example, in Ref. 63. In the case of considering a metallic particle whose size is much smaller than the wavelength, the calculation simplifies because it is sufficient to consider the quasistatic field solution, ${ }^{63}$ and the QD is also treated as a point dipole. It is well-known that the decay rate of an electric dipole in free space is

$$
\gamma_{0}=\frac{1}{2} \int_{S} \vec{E}^{0} \wedge \vec{H}^{0} \cdot d \vec{S} \propto \omega_{L}^{4}\left|\vec{d}_{0}\right|^{2},
$$

where $\vec{d}_{0}$ is the dipolar moment of the atom and $\vec{E}^{0}\left(\vec{H}^{0}\right)$ is the electric (magnetic) field in free space. When a nanoparticle is close to the atom, the radiation power will change since the electric and magnetic fields reflected on the particle act back upon the atom and modify the emission of the atomic dipole. In this new situation, the decay rate is

$$
\gamma=\frac{1}{2} \int_{S}\left(\vec{E}^{0}+\vec{E}^{R}\right) \wedge\left(\vec{H}^{0}+\vec{H}^{R}\right) \cdot d \vec{S} \propto \omega_{L}^{4}\left|\vec{d}_{t o t}\right|^{2},
$$

$\vec{E}^{R}\left(\vec{H}^{R}\right)$ being the reflected electric (magnetic) field, and $\vec{d}_{\text {tot }}$ being the total dipole moment of the hybrid system. In view of these considerations, we can compute an enhancement factor for the decay rate as $F_{e n h}=\gamma / \gamma_{0}=\left|\vec{d}_{t o t}\right|^{2} /\left|\vec{d}_{0}\right|^{2}$. This topic has been addressed in detail in previous works. ${ }^{63,64}$ The enhancement factor $\left(F_{\text {enh }}\right)$ depends on both the size and shape of the MNP and its relative orientation to the dipolar moments of the QD. The coherent-plasmonic field enhancement factor in the current case, $F_{e n h}^{j}$, reads as [see Eq. (11)]

$$
F_{\text {enh }}^{j} \equiv\left|\frac{\Omega_{j}}{\Omega_{j}^{0}}\right|^{2}=\left|1+\frac{S_{j} a^{3} \gamma\left(\omega_{L}\right)}{R^{3}}\right|^{2} \quad(j=1,2) .
$$

In view of the considerations raised when defining the enhancement factor in Eq. (16), the radiative decay rate of the atomic transitions modified by the coherent-plasmonic field enhancement are given by

$$
\gamma_{3 j}^{p}=\gamma_{3 j}^{(0)} F_{e n h}^{j} \quad(j=1,2)
$$

where the super-index 0 is used to indicate the values of the free space decay rates. A similar approach to estimate the modification of the decay rates has been used in other works (see, for example, Refs. 65-69). It is worth noting that depending on the orientation of the dipole moments of the QD emitter's transitions, the decay rates could become very different from one another, i.e., the value of $F_{\text {enh }}^{1}$ can strongly differ from that of $F_{e n h}^{2}$. This could result in an anisotropic Purcell factor enhancement which will have important consequences for controlling the time dynamics of this nano-hybrid system.

The equations of motion for the density matrix elements of the QD in the hybrid system and in an appropriated rotating frame can be derived from Eq. (10) and read 


$$
\begin{aligned}
\frac{\partial \rho_{31}}{\partial t}= & -\left\{\frac{\gamma_{31}^{p}+\gamma_{32}^{p}}{2}+i\left[\Delta_{31}-G_{1}\left(\rho_{11}-\rho_{33}\right)\right]\right\} \rho_{31}+i \Omega_{1}\left(\rho_{11}-\rho_{33}\right)+i\left(\Omega_{2}+G_{2} \rho_{32}\right) \rho_{21}, \\
\frac{\partial \rho_{21}}{\partial t}= & -\left[\frac{\gamma_{21}}{2}+i\left(\Delta_{31}-\Delta_{32}\right)\right] \rho_{21}+i\left(\Omega_{2}^{*}+G_{2}^{*} \rho_{32}\right) \rho_{31}-i\left(\Omega_{1}+G_{1} \rho_{31}\right) \rho_{23}, \\
\frac{\partial \rho_{32}}{\partial t}= & -\left\{\frac{\gamma_{31}^{p}+\gamma_{32}^{p}}{2}+i\left[\Delta_{32}-G_{2}\left(\rho_{22}-\rho_{33}\right)\right]\right\} \rho_{32}-i \Omega_{2}\left(\rho_{33}-\rho_{22}\right)+i\left(\Omega_{1}+G_{1} \rho_{31}\right) \rho_{12}, \\
\frac{\partial \rho_{33}}{\partial t}= & -\left(\gamma_{31}^{p}+\gamma_{32}^{p}\right) \rho_{33}+i\left(\Omega_{1}+G_{1} \rho_{31}\right) \rho_{13}-i\left(\Omega_{1}^{*}+G_{1}^{*} \rho_{13}\right) \rho_{31} \\
& +i\left(\Omega_{2}+G_{2} \rho_{32}\right) \rho_{23}-i\left(\Omega_{2}^{*}+G_{2}^{*} \rho_{23}\right) \rho_{32}, \\
\frac{\partial \rho_{22}}{\partial t}= & -\gamma_{21} \rho_{22}+\gamma_{32}^{p} \rho_{33}-i\left(\Omega_{2}+G_{2} \rho_{32}\right) \rho_{23}+i\left(\Omega_{2}^{*}+G_{2}^{*} \rho_{23}\right) \rho_{32},
\end{aligned}
$$

where $\Delta_{3 j}=\omega_{3 j}-\omega_{L}(j=1,2)$.

A close inspection of Eq. (18) reveals that the plasmonic interaction manifests in three ways: the first one relies on the enhancement of the Rabi frequencies which drive the QD according to the values given in Eq. (11). The second mechanism can be formally interpreted as a nonlinear frequency shift in the optical resonance causing a dynamical detuning. The value of the dynamical detuning for the optical coherence $\rho_{31}$ and $\rho_{32}$ are given by $\operatorname{Re}\left[G_{1}\left(\rho_{11}-\rho_{33}\right)\right]$ and $\operatorname{Re}\left[G_{2}\left(\rho_{22}-\rho_{33}\right)\right]$, respectively. In addition, the radiative decay rates are also modified by the plasmonic interaction as previously discussed. It should be remarked that the three above mentioned mechanism strongly depend on the QD-MNP distance $R$ and its size through $a$.

The electric field radiated by the hybrid system in the THz regime $\left(E_{R}(t)\right)$ is proportional to the second temporal derivative of the coherent time-varying polarization $P \propto \rho_{21}(t)$, i.e., $E_{R}(t) \propto \partial^{2} \rho_{21}(t) / \partial t^{2}$. In what follows we will analyze the effects of localized surface plasmons on the radiated $\mathrm{THz}$ signal.

\section{NUMERICAL SIMULATIONS AND DISCUSSION}

We consider a spherical gold MNP with radius $a=8-10 \mathrm{~nm}$. The dielectric constant for gold is obtained by interpolating the tabulated experimental data from Ref. 70 . We use the following parameters to describe the QD molecule: $\epsilon_{s}=\epsilon_{B}=12.96, \hbar \omega_{13}=1.526 \mathrm{eV}$, an energy splitting of $\hbar \omega_{21}=6 \mathrm{meV}$, a relaxation time of $\gamma_{31}^{(0)}=\gamma_{32}^{(0)} \equiv \gamma_{0}$ $=0.17 \mathrm{ps}^{-1}$, and dipole moments $\mu_{23}=\mu_{13}=31.2 \mathrm{D}$. These are typical values for the decay rates and the dipolar moments commonly used. The choosing of lowers' level splitting of $6 \mathrm{meV}$ is close to the value reported in Ref. 59 of $1.1 \mathrm{meV}$. The specific value of $\hbar \omega_{21}$ is not essential to the purposes of the current work, and merely manifest as a change in the frequency of the $\mathrm{THz}$ generated signal.

The lowers' level dephasing is set to $\gamma_{21}=0.3 \gamma_{0}$. We consider that the system is driven by either a single Gaussian laser pulse centered around $814 \mathrm{~nm}$ or by a finite train of identical delayed pulses. The pulse/s can be derived from a Ti:sapphire laser. In the case of a single laser pulse, it can be written as $\Omega_{1}^{0}(t)=\Omega_{2}^{0}(t)=\frac{\Omega_{0}}{\sqrt{2 \pi \sigma^{2}}} e^{-\left(t-t_{p}\right)^{2} / 2 \sigma^{2}}$, where $\sqrt{2} \sigma$ is the $1 / e$ half-width, $\Omega_{0}$ is the normalized (dimensionless) peak amplitude which measures the pulse area, and $t_{p}$ is the center of the pulse. The bandwidth of the laser pulse is assumed to be large enough to be able to drive the two excitonic transitions simultaneously. The temporal pulse length is $\sigma=0.05 / \gamma_{0} \approx 300 \mathrm{fs}$. Finally, the polarization parameters are $S_{1}=S_{2}=-1$.

We numerically solve the density matrix equations of motion [Eq. (18)] using a time interval $\left[0, t_{f}\right]$. The instant of time $t_{f}=200 / \gamma_{0} \approx 1.2 \mathrm{~ns}$ is selected to be large enough to allow for the proper sampling of the low frequency components of the radiated field which account for the terahertz signal $E_{R}(t)$. For all simulations, we consider $\rho_{11}(0)=1$, and $\rho_{i j}(0)=0(i, j=1,2,3, i$ and $j \neq 1)$.

Figure 2(a) displays the time evolution of the electric field of the terahertz signal in the absence (dashed line) and in the presence (solid line) of the MNP. The distance from the QD to the MNP was set to $R=15 \mathrm{~nm}$ and the radius of the MNP was selected to be $a=8 \mathrm{~nm}$. It becomes clear that the presence of the MNP leads to a larger terahertz signal than the one obtained in its absence. This effect is further confirmed when analyzing the power spectrum of the signals depicted in panel (a) and they are displayed in panel (b). The maximum of the power spectrum is reached at a frequency $\nu_{c} \approx 1.45 \mathrm{THz}$ (vertical dashed line) which is roughly the same value as the lowers' level splitting. The enhancement of the peak value in the power spectrum of the terahertz signal can be attributed to the dipole-dipole interaction between the QD and the MNP. Let us analyze in detail which is the major mechanism for causing the terahertz signal enhancement. The values of the nonlinear parameters are $G_{1}=G_{2} \approx(-0.17+i 0.37) \gamma_{0}$. Note that both the real and imaginary parts of the nonlinear parameters remain lower than one in units of $\gamma_{0}$, which indicates us that the energy transfer from the QD to the MNP and the dynamical detuning should have a negligible effect in the time dynamics of the hybrid system and, consequently, in the generation of the terahertz signal. In view of this, the influence of the MNP on the QD reduces to the enhancement of the effective Rabi frequency $\left(\left|\Omega_{j}\right| \approx 3.78\left|\Omega_{j}^{0}\right|\right)$ which drives the excitonic transitions. This effect is accompanied by the modification of the optical decay rates: $\gamma_{31}^{p}=\gamma_{32}^{p} \approx 14.3 \gamma_{0}$. In order to clarify the role of these two mechanisms, we plot in panel (c) the $\mathrm{THz}$ signal without considering the change of the decay rates 

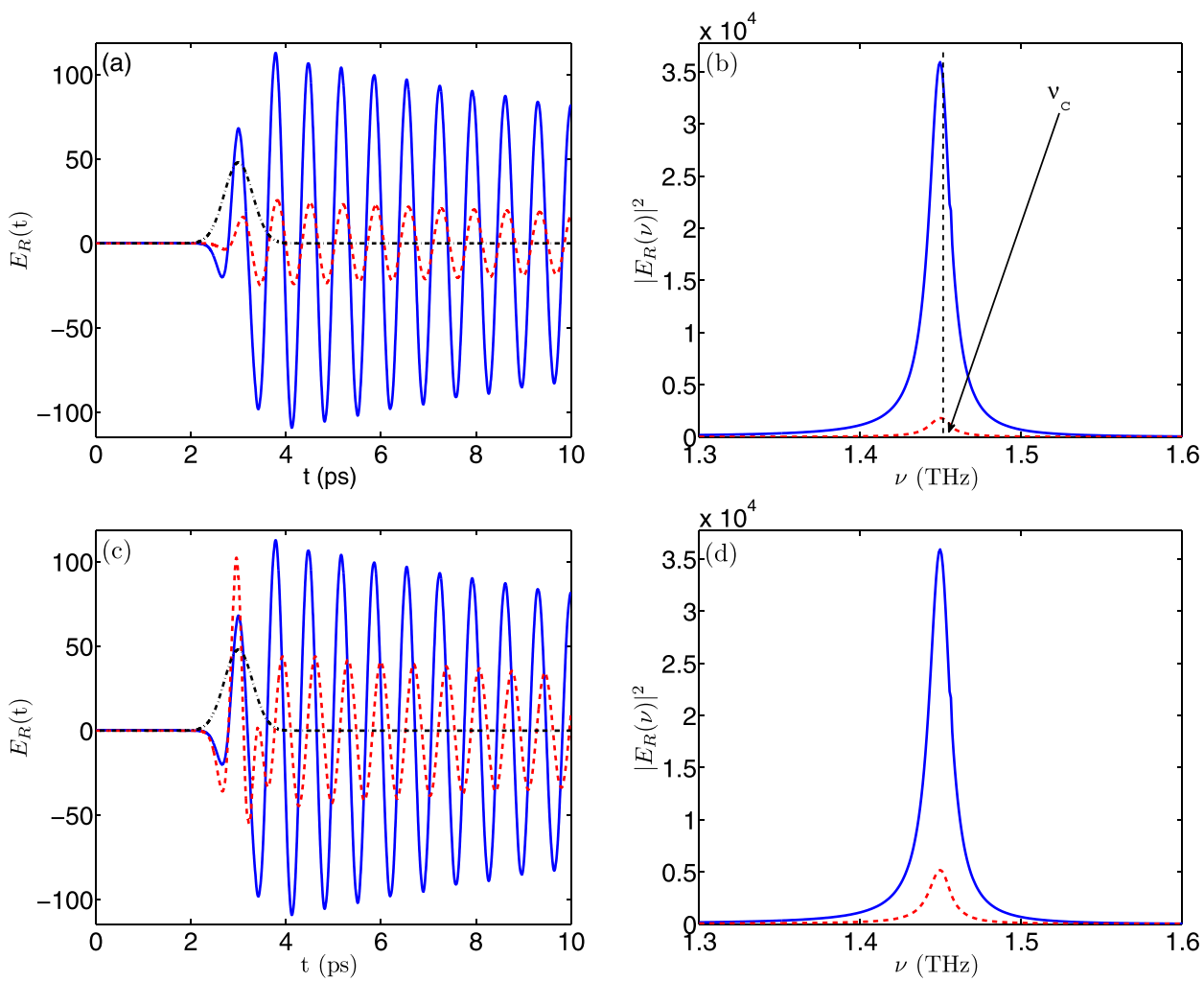

FIG. 2. (a) The terahertz radiation signals $\left(E_{R}(t)\right)$ emitted after irradiating the hybrid system with a femtosecond pulse at resonance with the $|1\rangle \leftrightarrow|3\rangle$ optical transition $\left(\Delta_{31}=0\right)$, in the absence (dashed line)/presence(solid line) of the MNP. (b) The power spectrum of the respective signals $\left(\left|E_{R}(\nu)\right|^{2}\right.$ ) displayed in panel (a). (c) The terahertz radiation signals $\left(E_{R}(t)\right)$ emitted after irradiating the hybrid system with a femtosecond pulse like in panel (a) considering/neglecting the modification of the decay rates of the optical transitions (solid/dashed line). (d) The power spectrum of the respective signals $\left(\left|E_{R}(\nu)\right|^{2}\right)$ displayed in panel (c). In all panels the pulse center is $t_{p} \approx 3 \mathrm{ps}$, its width is $\sigma \approx 0.3 \mathrm{ps}$, the pulse area is $\Omega_{0}=0.5$. The MNP has a radius of $a=8 \mathrm{~nm}$ and it is located $R=15 \mathrm{~nm}$ apart from the QD. The dashed-dotted line in (a) and (c) stands for the scaled driving pulse. due to QD-MNP interaction (dashed line), and using the same parameters as in panel (a). For comparison purpose, we also plot the $\mathrm{THz}$ signal when such modification of the decay rates is taken into account (solid line). This figure reveals that the change of the decay rates due to the QD-MNP interaction results in a dramatic change of the time evolution of the radiated field while the driving pulse is on (dashed-dotted line). In addition, disregarding the modification of the decay rates results in a low terahertz signal (dashed line). This effect can be confirmed by inspecting panel (d) which shows the power spectrum of the signals of panel (c). In summary, the MNP produces a huge enhancement of the $\mathrm{THz}$ signal. This result can be attributed to the enhancement of both the Rabi frequencies and the optical decay rates.

The system dynamics is expected to depend also on the detuning $\Delta_{31}$. Experimentally, $\Delta_{31}$ is controlled by varying the frequency of the driving laser. Thus, we now focus our attention on the peak value of the power spectrum of the signal $\left(\left|E_{R}\left(\nu_{c}\right)\right|^{2}\right)$. We performed numerical simulations by varying both the pulse area $\left(\Omega_{0}\right)$ and the detuning $\left(\Delta_{31}\right)$. The resulting map is displayed in Fig. 3 , indicating that a huge enhancement of the terahertz signal is obtained for a wide pairs of values $\left(\Delta_{31}, \Omega_{0}\right)$. This indicates that such enhancement can be achieved without requiring precise control for the optical detuning, while the peak value at $\nu_{c}$ can be tuned by a proper selection of the pulse area. This point is essential to address the experimental implementation of this hybrid system.

Figure 3 has shown that the area of the incident pulse is an external parameter which allows for the control of the terahertz signal. In addition, the magnitude of the enhancement of the effective Rabi frequency (see Eq. (11)) is explicitly dependent on the size of the MNP. Thus, we resort to analyze the influence of the pulse area $\left(\Omega_{0}\right)$ and the size of the MNP on the generated signal. Figure 4(a) displays the peak value of the power spectrum at $\nu_{c}\left(\left|E_{R}\left(\nu_{c}\right)\right|^{2}\right)$ as a function of the pulse area $\Omega_{0}$. In the case of an isolated QD (dashed line), we observe an oscillatory behavior in the achieved peak value at $\nu_{c}$. The period of the oscillations is shortened in the presence of the MNP and an enhancement of the peak value is achieved (dashed-dotted line). This shortening appears due to the strong exciton-plasmon coupling, which produces a huge increase of the effective Rabi frequency as indicated in Eq. (11). The change of the radius of the MNP from $a=8 \mathrm{~nm}$ (dashed-dotted line) to $a=10 \mathrm{~nm}$ (solid line) results in a further increase of the peak value of the terahertz signal

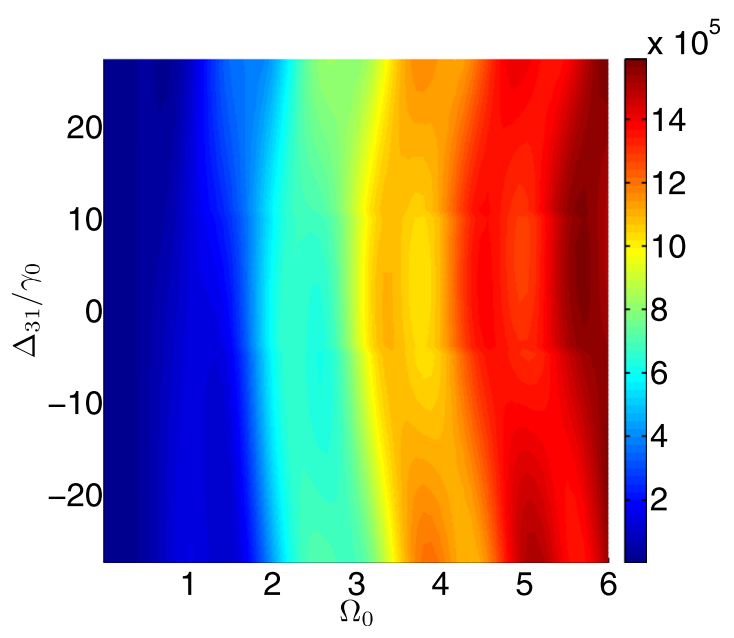

FIG. 3. Peak value of the power spectrum of the terahertz signal $\left(\left|E_{R}\left(\nu_{c}\right)\right|^{2}\right)$ as a function of the pulse area $\Omega_{0}$ and the optical detuning $\Delta_{31}$. The rest of parameters as in Fig. 2. 

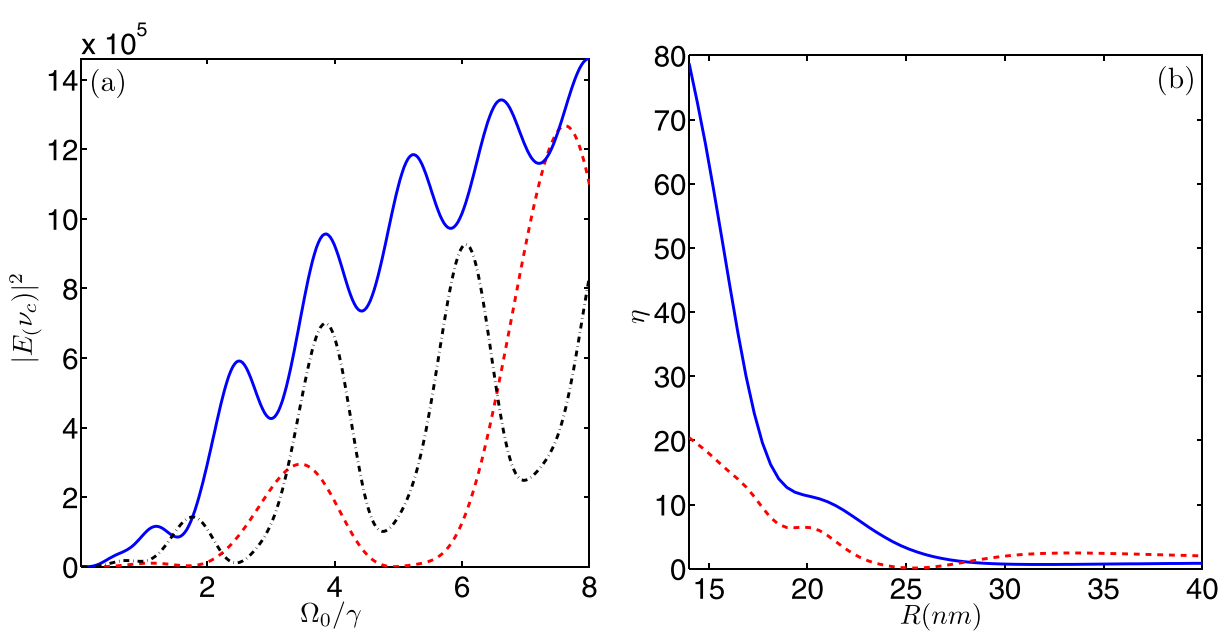

FIG. 4. (a) Peak value of the power spectrum of the THz field $\left(\left|E_{R}\left(\nu_{c}\right)\right|^{2}\right)$ as a function of the pulse area $\Omega_{0}$. Isolated QD (dashed line) and hybrid system: $(R=20, a=10) \mathrm{nm}$ (solid line) and $(R=20, a=8) \mathrm{nm}$ (dashed-dotted line). (b) Enhancement factor $\eta$ as a function of the distance $R$ in the case of $\Omega_{0}=2.5$ (dashed line) and $\Omega_{0}=1.2$ (solid line). The radius of the MNP is $a=10 \mathrm{~nm}$. The rest of parameters as in Fig. 2. accompanied by an additional shortening of the period of oscillation. This behavior can be explained by taking into account the fact that the plasmonic interaction enhances the effective Rabi frequency and also produces a modification of the decay rates $\left(\gamma_{3 j}^{p}, j=1,2\right)$ due to the term which scales with the cube of the radius of the MNP (see Eq. (16)). The curve with the largest radius of the MNP (solid line) is of special interest since it allows to obtain a moderate peak amplitude even for modest values of the pulse area $\Omega_{0} \approx 2.5$. This is of special interest for the experimentalists since it does not require the use of a very high energy driving pulse.

Let us define an enhancement factor $(\eta)$ as the quotient of the power of the terahertz signal at $\nu_{c}$ obtained in the presence of the MNP $\left(\left|E_{w}\left(\nu_{c}\right)\right|^{2}\right)$ to that obtained for the isolated QD $\left(\left|E_{\text {iso }}\left(\nu_{c}\right)\right|^{2}\right.$ ), i.e., $\eta \equiv\left|E_{w}\left(\nu_{c}\right)\right|^{2} /\left|E_{\text {iso }}\left(\nu_{c}\right)\right|^{2}$. Figure 4(b) presents the magnitude $\eta$ versus the distance $R$ for two values of the pulse area. These curves indicate us that for an adequate selection of the distance $R$ and the pulse area $\Omega_{0}$, up to an eightyfold enhancement can be achieved. Note that for a separation larger than $R=40 \mathrm{~nm}$ the enhancement is reduced, since the dipole-dipole interaction is negligible at such large distances: $\Omega_{j} \approx \Omega_{j}^{0}$ and $\gamma_{31}^{p} \approx \gamma_{31}^{(0)}$.

Now we turn our attention to the possibility of obtaining a precise control of the duration and the shape of the generated terahertz signals. Different schemes have been considered to shape the terahertz electromagnetic waves. Such coherent optical control over individual quantum systems in semiconductors has been the subject of active research over the past decade ${ }^{71}$ because of its potential applications in atom optics, ${ }^{72}$ preparation of entanglement, ${ }^{73}$ and quantum computation. ${ }^{74,75}$ Three main strategies, that is, temporal coherent control, optimal control, and adiabatic passage, have been proposed to realize quantum coherent control. $^{76}$ Stimulated Raman adiabatic passage (STIRAP) $)^{71,77,78}$ has emerged as a very efficient and robust way to achieve complete coherent population transfer between two discrete states in an atomic or a molecular system. This technique has played a major role in population transfer in $\Lambda$ and ladder systems. ${ }^{71,79,80}$ In what follows we will analyze the use of a train of pulses which drive simultaneously the two optical transitions or a train of delayed pulses in an STIRAP schema.

Here, we will analyze how the application of two delayed pulses which drive simultaneously the two optical transitions would allow to improve the efficiency of the terahertz signal generation. To this end, we carried out numerical simulations to compare the terahertz signal obtained when a single driving pulse is applied with that obtained under the application of two consecutive pulses of the same width. The two-pulse train is given by $\Omega_{1}^{0}(t)=\Omega_{2}^{0}(t)=\frac{1}{2} \frac{\Omega_{0}}{\sqrt{2 \pi \sigma^{2}}}$ $\left(e^{-\left(t-t_{p}\right)^{2} / 2 \sigma^{2}}+e^{-\left(t-t_{p}-T_{r}\right)^{2} / 2 \sigma^{2}}\right)$. The second pulse is delayed by $T_{r}=1 / \nu_{21}$, and $\nu_{21}$ being the frequency associated to the lowers' level splitting. In the case of the two pulses, the area of the two-pulse train was selected to be identical to the area used in the single driving pulse, which is guaranteed by the prefactor $1 / 2$. Note that in both cases, the single pulse and the two-pulse train cases, the laser field drives simultaneously both optical transitions. In the simulations depicted in Fig. 5, we have chosen the width of each one of the driving pulses to be lesser than in the previous figures of this work. This choice is motivated by the fact that the time interval between the centers of two consecutive pulses must be larger than the width of each one of them, which allows to drive the optical transitions with non-overlapping pulses.

Figure 5(a) displays the terahertz radiation signals emitted by irradiation with a single femtosecond pulse tuned to resonance (dashed line), centered at $t_{p} \approx 3 \mathrm{ps}, \sigma \approx 60 \mathrm{fs}$ and $\Omega_{0}=0.75$. The resulting terahertz signal obtained for the case of two-pulse excitation is also shown (solid line). We can observe that the application of a second pulse allows to manage the terahertz radiation signal. In particular, the amplitude of the $\mathrm{THz}$ is enhanced by a factor greater than 2 when compared to the signal obtained in the single pulse excitation case. Panel (b) shows the power spectrum of the respective $\mathrm{THz}$ signals obtained in both situations. Again we observe that the terahertz field has the maximum peak at a frequency close to $\nu_{21} \simeq 1.45 \mathrm{THz}$, which demonstrates the enhancement arising from the use of two pulses. The particular choice of the delay between the two pulses $\left(T_{r}=1 / \nu_{21}\right)$ is motivated by the fact that this time interval is related to the beating time of the lowers' level coherence. Figure 5(c) displays the peak value of the power spectrum $\left(\left|E_{R}\left(\nu_{c}\right)\right|^{2}\right)$ versus the time delay between the two pulses $\left(T_{r}\right)$ in the presence/absence of the MNP (solid/dashed line). The vertical dashed line stands for the value $T_{r}=1 / \nu_{21}$. This panel reveals that the particular choice used to produce panel (a) 

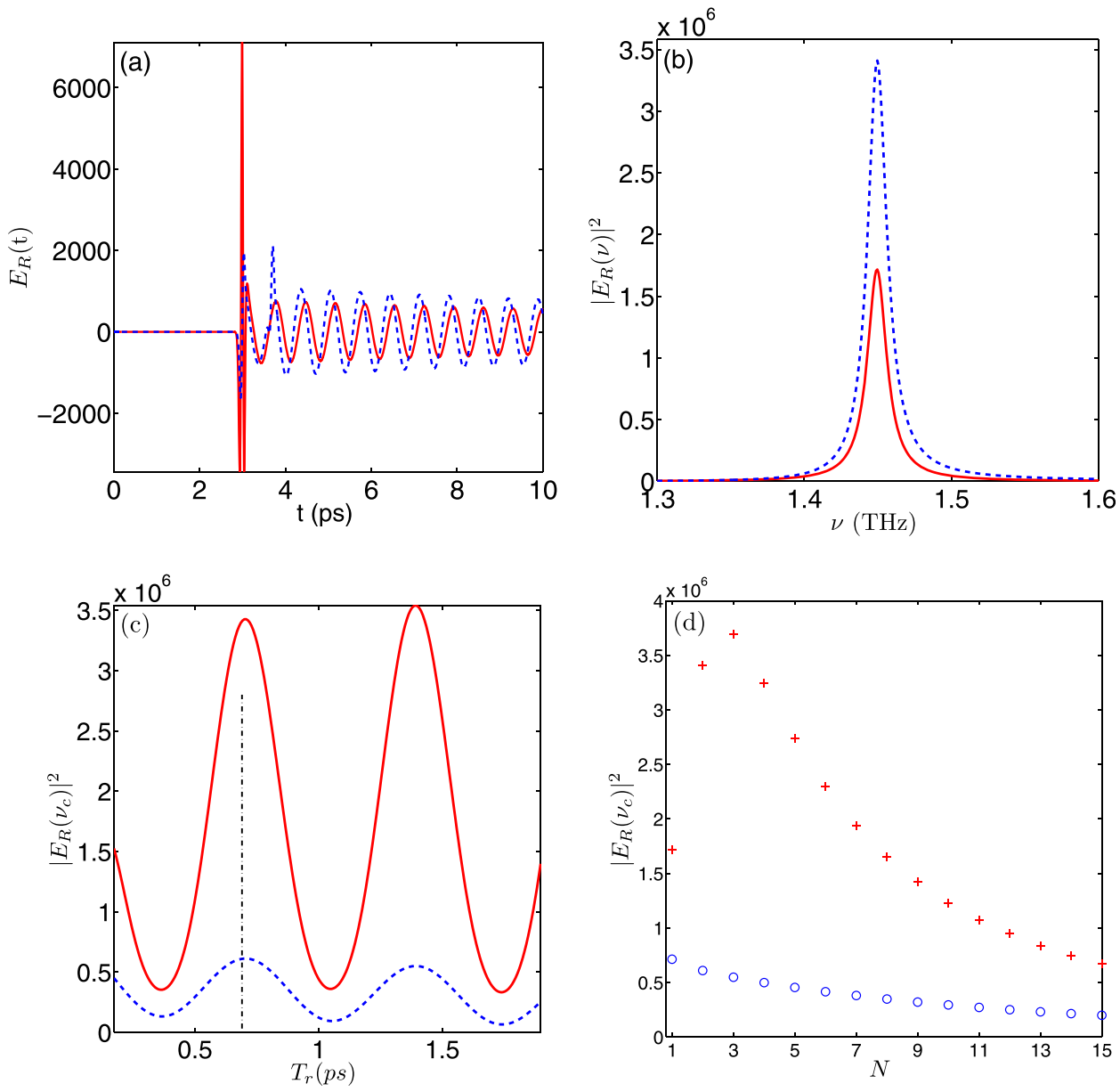

FIG. 5. (a) The terahertz radiation signals for pumping wave forms emitted by irradiation with a single femtosecond pulse (solid line) at $\Delta_{31}=0$ centered at $t_{p} \approx 3 \mathrm{ps}$ and width $\sigma \approx 60 \mathrm{fs}$ (solid line) and two pulses of the same width and delayed $T_{r}=1 / \nu_{21}$ (dashed line). (b) The power spectrum of the respective signals $\left(\left|E_{R}(\nu)\right|^{2}\right)$ displayed in panel (a). (c) Peak amplitude of $\mathrm{THz}$ field $\left(\left|E_{R}\left(\nu_{c}\right)\right|^{2}\right)$ versus the time delay between the two pulses $\left(T_{r}\right)$ in the absence/presence of the MNP (dashed/solid line). (d) Peak amplitude of $\mathrm{THz}$ field $\left(\left|E_{R}\left(\nu_{c}\right)\right|^{2}\right)$ as a function of the number of pulses $N$ in the absence $(0)$ and in the presence $(+)$ of the MNP for $T_{r}=1 / \nu_{12}$. In all panels the MNP is set at a distance $R=15 \mathrm{~nm}$, and $\Omega_{0}=0.75$. maximizes the enhancement of the terahertz signal in the two-pulse train. The oscillatory behavior of the two curves indicates that the use of time delay close to integer multiples of $1 / \nu_{21}$ allows for the maximization of the peak value of the terahertz signal.

Finally, let us consider the case where both transitions are driven simultaneously by a finite train of identical pulses of

the form $\Omega_{1}^{0}(t)=\Omega_{2}^{0}(t)=\frac{1}{N} \frac{\Omega_{0}}{\sqrt{2 \pi \sigma^{2}}} \sum_{j=1}^{N} e^{-\left(t-t_{p}-(j-1) / \nu_{21}\right)^{2} / 2 \sigma^{2}}$. Figure 5(d) displays the peak amplitude of the $\mathrm{THz}$ field $\left(\left|E_{R}\left(\nu_{c}\right)\right|^{2}\right)$ as a function of the number of pulses $(N)$ in the presence/absence of the MNP $(+/ 0)$. In the case of an isolated QD (o), we found a monotonic decrease of the peak value achieved as the number of pulses increases. This situation is dramatically modified by the presence of the MNP: a close inspection of panel (d) reveals that there exists an optimum value of the number of pulses $\left(N_{\text {opt }}=3\right)$ which maximizes the peak value of the power spectrum of the signal. This is of particular interest for the experimentalists since the use of finite train of pulses allows to obtain up to a nearly sevenfold enhancement of the signal by using pulses of small area compared to the case of using a single pulse of large area. We would like to remind here that the simulation was performed by imposing that the area of the single driving pulse is identical to the total area of the finite multipulse train.

Now we turn our attention to the use of a pair of delayed pulses which drive the two optical transitions in an STIRAP schema, in order to analyze whether this choice would allow for a high terahertz signal. To this end, we carried out numerical simulations to compare the terahertz signal obtained when a pair of simultaneous pulses of the same width drives the two optical transitions of the form $\Omega_{1}^{0}(t)=\Omega_{2}^{0}(t)$ $=\frac{\Omega_{0}}{\sqrt{2 \pi \sigma^{2}}} e^{-\left(t-t_{p}\right)^{2} / 2 \sigma^{2}}$, with the case of using non-simultaneous pulses for both optical transitions given by $\Omega_{1}^{0}(t)=\frac{\Omega_{0}}{\sqrt{2 \pi \sigma^{2}}}$ $e^{-\left(t-t_{p}\right)^{2} / 2 \sigma^{2}}$ and $\Omega_{2}^{0}(t)=\frac{\Omega_{0}}{\sqrt{2 \pi \sigma^{2}}} e^{-\left(t-t_{p}+\delta \tau\right)^{2} / 2 \sigma^{2}}, \delta \tau$ being the time separation between the time center of the two pulses. We allow $\delta \tau$ to be either positive/negative which allows to simulate the driving of the optical transitions in an counterintuitive/intuitive way. It is worth mentioning that the use of driving fields in a counterintuitive way, i.e., where the pulse which drives the $|2\rangle \leftrightarrow|3\rangle$ optical transition precedes the pulse which drives the $|1\rangle \leftrightarrow|3\rangle$ transition, has been proved to be more useful for the optimum transfer of population between the two lower levels in a similar $\Lambda$-like atom as the one considered here (see, for example, Refs. 71, 79, and 80). In both cases, the area of the pulses used was the same. Figure 6(a) displays the terahertz radiation signals emitted by irradiation with a single femtosecond pulse tuned to resonance which drive simultaneously the two optical transitions (solid line), centered at $t_{p}=0.3 \mathrm{ps}, \sigma \approx 300 \mathrm{fs}$, and $\Omega_{0}=2$. The resulting terahertz signal obtained for the case of two pulse excitation and delayed $\delta \tau=\sigma$ is also shown (dashed line). We can observe that the application of a 

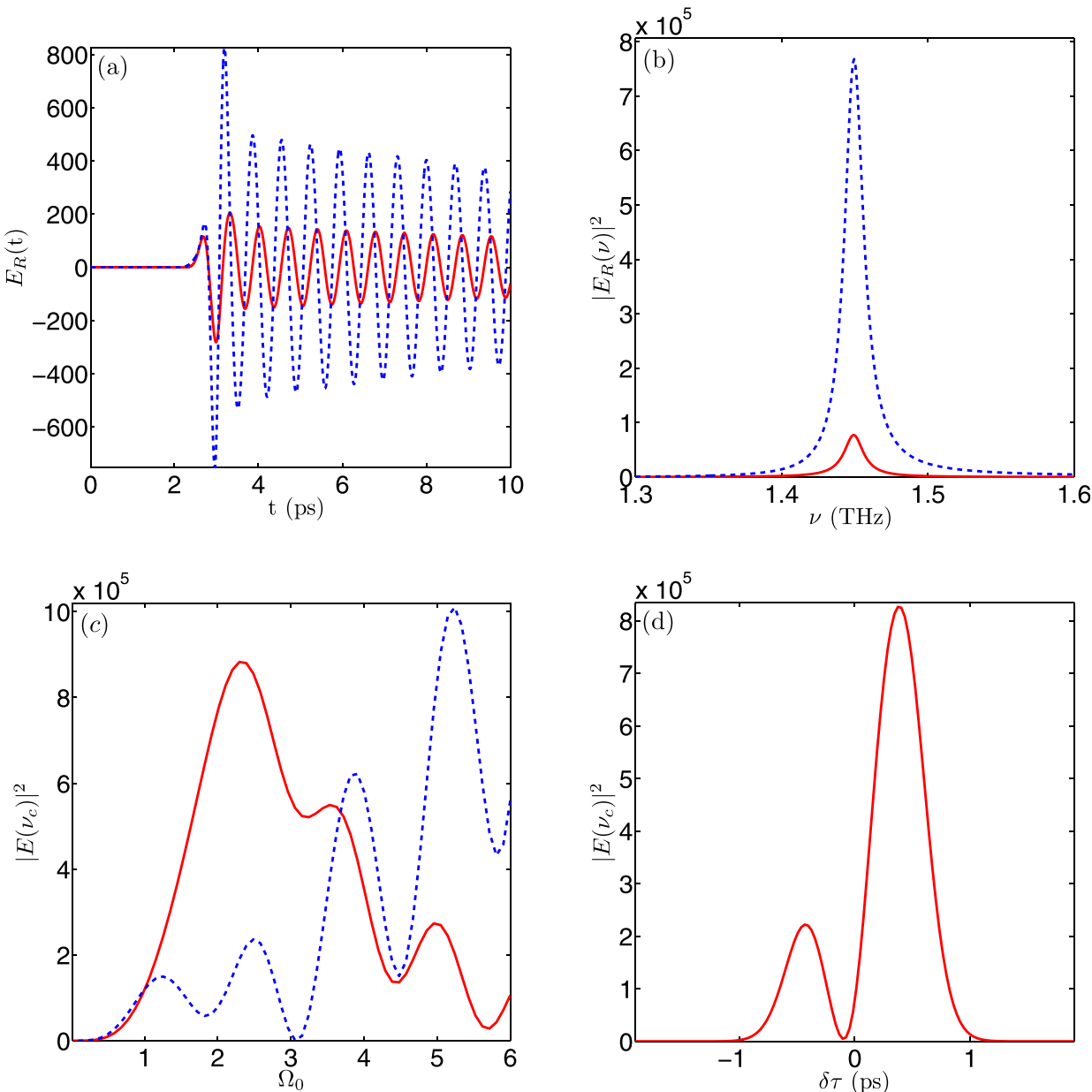

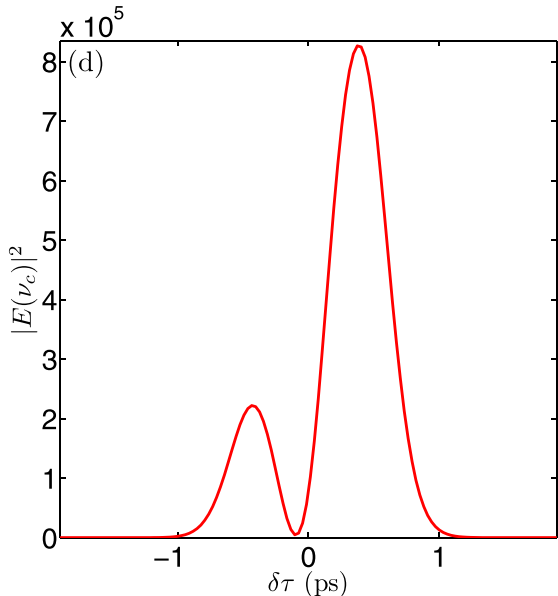

FIG. 6. (a) The terahertz radiation signals $\left(E_{R}(t)\right)$ emitted after irradiating the hybrid system with two simultaneous/non-simultaneous femtosecond pulses in a STIRAP like sequence (solid line/dashed line). The delay is equal to the width of each individual pulse $\delta \tau=300$ fs. (b) The power spectrum of the respective signals $\left(\left|E_{R}(\nu)\right|^{2}\right)$ displayed in panel (a). The MNP has a radius of $a=8 \mathrm{~nm}$ and is located at $R=15 \mathrm{~nm}$, each train of pulses have the same area $\Omega_{0}=2$, and the pulses have the same temporal width $\sigma=300$ fs. (c) Peak value of the power spectrum $\left(\left|E_{R}\left(\nu_{c}\right)\right|^{2}\right)$ versus the Rabi frequency $\Omega_{0}$ for two simultaneous $\quad(\delta \tau=0) /$ non-simultaneous $(\delta \tau=300 \mathrm{fs})$ femtosecond pulses (dashed/solid line), and other parameters like in panel (a). (d) Peak value of the power spectrum of the terahertz signal $\left(\left|E_{R}\left(\nu_{c}\right)\right|^{2}\right)$ as a function of the pulse delay $(\delta \tau)$, using the rest of data as in panel (a) non-simultaneous second pulse in a STIRAP way allows to produce a huge enhancement of the terahertz radiation signal. In particular, the amplitude of the $\mathrm{THz}$ signal is enhanced by a factor greater than 9.9 when compared to the signal obtained in the simultaneous excitation case. Panel (b) shows the power spectrum of the respective $\mathrm{THz}$ signals obtained in both situations. Again we observe that the terahertz field has the maximum peak at a frequency close to $\nu_{21}$ $\simeq 1.45 \mathrm{THz}$. Panel (c) shows the peak value of the power spectrum at $\nu_{c}$ versus the Rabi frequency $\Omega_{0}$ in the case with two simultaneous/non-simultaneous pulses. It is remarkable that the use of the STIRAP schema for driving the optical transitions produce a large peak value than that obtained in the simultaneous driving in the range from low to intermediate values of the pulse area $\left(0<\Omega_{0} \leq 3\right)$, whereas for larger areas the STIRAP schema shows a worse performance. This is of interest for the experimental testing of these predictions, since the use of large areas would require more powerful lasers to drive the hybrid system. Let us define an enhancement factor $\epsilon \equiv \frac{\left|E_{R}\left(\nu_{c}, \delta \tau \neq 0\right)\right|^{2}}{\left|E_{R}\left(\nu_{c}, \delta \tau=0\right)\right|^{2}}$. This will allow to know the relative efficiency of the STIRAP-like driving schema compared to the simultaneous excitation one. Magnitude $\epsilon$ ranges from 1 to 3 in the interval $\Omega_{0} \in[1,2.5]$ and exhibits a maximum of 11.3 at $\Omega_{0}=1.9$, indicating the existence of an optimum value of the pulse area to enhance the $\mathrm{THz}$ signal. Finally, panel (d) depicts the peak amplitude of the power spectrum of the $\mathrm{THz}$ signal at $\nu_{c}$ versus the time delay $\delta \tau$. Positive values of $\delta \tau$ correspond to the counterintuitive driving STIRAP schema, whereas negative values of $\delta \tau$ match to the intuitive driving schema, where the pulse which drives the $|1\rangle \leftrightarrow|3\rangle$ optical transition precedes the pulse which drives the $|2\rangle \leftrightarrow|3\rangle$ transition. The maximum attained in the counterintuitive driving schema is fourfold greater than the maximum obtained in the intuitive driving one.

In order to get some physical insight about the numerical results, we resort to solve a linearized version of Eq. (18). We assume that each pulse duration is sort compared with the system response times, and the period of the quantum beat, so that the optical fields can be described by impulsive functions ${ }^{81}$

$$
\hat{E}(t)=\sum_{0}^{N-1} \mathcal{E}\left(t-n T_{r}\right) e^{-i\left(\omega_{L} t-n \omega_{L} T_{r}\right)},
$$

where $\mathcal{E}(t)$ is the envelope function and $T_{r}$ is the time between pulses.

We are interested in obtaining the response of the sublevels coherence $\rho_{21}$ after a long time interaction with the pulse train as the system reaches the steady state regime. In the weak pulse limit, we can neglect the variations in the populations and then write $\rho_{11}=\rho_{11}^{f}$ and $\rho_{22}=\rho_{22}^{f}$, where super-index $f$ indicates the steady-state values of the corresponding magnitude. In the frequency domain and in the weak field limit, the atomic coherences can be derived from Eq. (18) and read 


$$
\begin{aligned}
-i \omega \rho_{31}(\omega)= & -\left(\gamma_{31}^{p}+i\left(\omega_{31}-G_{1}\right)\right) \rho_{31}(\omega) \\
& +i \Omega_{1}(\omega) \rho_{11}^{f}+i \Omega_{2}(\omega) \otimes \rho_{21}(\omega), \\
-i \omega \rho_{23}(\omega)= & -\left(\gamma_{32}^{p}-i\left(\omega_{32}-G_{2}\right)\right) \rho_{23}(\omega) \\
& +i \Omega_{2}^{*}(\omega) \rho_{22}^{f}-i \Omega_{1}^{*}(\omega) \otimes \rho_{21}(\omega), \\
-i \omega \rho_{21}(\omega)= & -\left(\gamma_{21}+i \omega_{21}\right) \rho_{21}(\omega) \\
& +i \Omega_{2}^{*}(\omega) \rho_{31}-i \Omega_{1}(\omega) \otimes \rho_{21}(\omega),
\end{aligned}
$$

where $\Omega_{j}(\omega)$ stands for the Fourier transform of the pulse train given by ${ }^{81}$

$$
\Omega_{j}(\omega)=\frac{2 \pi}{T_{r}} \frac{\mu_{3 j} \mathcal{E}\left(\omega-\omega_{L}\right)}{\hbar} \sum_{m=-\infty}^{\infty} \delta\left(\omega-\omega_{m}\right) j=1,2,
$$

with $\omega_{m}=\frac{2 m \pi}{T_{r}}$. In the above equations, $\Omega_{j}(\omega) \otimes \rho_{i j}(\omega)$ is the convolution between the two functions. Using Eq. (21) and the convolution theorem, we can write

$$
\begin{aligned}
\Omega_{j}(\omega) \otimes \rho_{i j}(\omega) & =\frac{2 \pi}{T_{r}} \frac{\mu_{3 j} \mathcal{E}\left(\omega-\omega_{L}\right)}{\hbar} \sum_{m=-\infty}^{\infty} \delta\left(\omega-\omega_{m}\right) \otimes \rho_{i j}(\omega) \\
& =\bar{\Omega}_{j}(\omega) \otimes \rho_{i j} \sum_{m} \rho_{i j}\left(\omega-\omega_{m}\right)
\end{aligned}
$$

Pluging this result in Eq. (20), we can obtain the coherence between the two lower levels as

$$
\rho_{21}(\omega) \simeq \frac{\overline{\Omega_{1}^{0}} \overline{\Omega_{2}^{0}}\left|1+\frac{S_{1} a^{3} \gamma(\omega)}{R^{3}}\right|^{2}}{\left[\omega-\omega_{21}-i \gamma_{21}\right]} \sum_{m, m^{\prime}}\left(\frac{\rho_{11}^{f}}{\left[\omega-\omega_{31}+\omega_{m}-i\left(\gamma_{31}^{p}-i G_{1}\right)\right]}-\frac{\rho_{22}^{f}}{\left[\omega-\omega_{32}-\omega_{m^{\prime}}-i\left(\gamma_{32}^{p}-i G_{2}\right)\right]}\right) \delta\left(\omega-\omega_{m}+\omega_{m^{\prime}}\right) .
$$

A close inspection of Eq. (23) allows us to explain the origin of the enhancement of the $\mathrm{THz}$ signal by the presence of the MNP. In this equation, we can devise that the lowers' level coherence is proportional to the product of the Rabi frequencies of the driving fields modified by the distance dependent term appearing in Eq. (11). In addition, we obtain a combination of several terms corresponding to one-photon resonance, as indicated by the denominators $\omega-\omega_{31}+\omega_{m}-$ $i\left(\gamma_{31}^{p}-i G_{1}\right)$ and $\omega-\omega_{32}-\omega_{m^{\prime}}-i\left(\gamma_{32}^{p}-i G_{2}\right)$. These terms incorporate the distance dependence of the decay rates by the plasmonic interaction and the Förster terms $\left(G_{j}, j=1,2\right)$. These facts provide a qualitative explanation of the numerical results found in Figs. 2-5. Finally, Eq. (23) also indicates us that the lowers' level coherence exhibits peaks at $\omega=\omega_{m^{\prime}}-\omega_{m}$. Among these peaks, there exists one which exhibit a resonant behavior when $\omega \approx \omega_{21}$. This finding explains the appearance of maxima at multiples of $T_{r}=1 / \nu_{21}$ in panel (c) of Fig. 5.

\section{CONCLUSIONS}

In this work, we present a description of the excitonplasmon interaction in a QD-MNP hybrid system intended for terahertz generation. The QD molecule is modeled as a three level-like atomic system of the $\Lambda$-type and the MNP is considered to be a nanosphere. The exciton-plasmon interaction manifest in two ways: the localized surface charge oscillations in the MNP modify the decay rates of the QD depending on the distance $R$, and it produces and enhancement of the effective Rabi frequency experienced by the QD. The terahertz radiation signal arises from the coherent oscillation of the lowers' level coherence and is dramatically modified by the presence of the MNP. We have shown that the amplitude of the obtained terahertz signal depends on the area of the optical excitation pulse, the distance $R$, and the size of the MNP. Coherent control of quantum beats in the current hybrid system is demonstrated by using two or multiple delayed pumping pulses in either a simultaneous excitation of the two optical transitions and in an STIRAP-like pumping schema. These results will be very useful for the pulse shaping of a terahertz signal generated by optically pumping a QD-MNP nano-composite.

Finally, it should be stated that, although speculative, the hybrid system here investigated can be implemented in realistic QD-MNP systems. Recently, the possibility to synthesize on demand light transients have been experimentally demonstrated. ${ }^{82}$ The light pulses can be used to probe fine details of atomic-scale electron-hole motion and its coherent oscillations in the current hybrid system. The present state of the art of sample growth and coherent-carrier control allows the implementation of QDs with the optical characteristics like the one considered here. Thus, $\Lambda$-like systems in coupled semiconductor quantum dots have been theoretically proposed $^{58}$ and realized ${ }^{59}$ with lower levels' splittings similar to the one considered in this work. In addition, hybrid structures consisting of self-assembled QDs have been grown and covered with metal nanocrystals. ${ }^{83}$ We would like to draw the attention to very recent works where the controlled coupling of a single epitaxial QD to a plasmonic nano-antenna has been demonstrated ${ }^{84,85}$ Thus, the structure modeled here could be fabricated using available nanotechnologies for the growth and the precise positioning of the involved elements.

\section{ACKNOWLEDGMENTS}

This work has been supported by Project No. FIS201022082 (MICINN) from Spain. 
${ }^{1}$ H. Ito, F. Nakajima, T. Furuta, and T. Ishibashi, Semicond. Sci. Technol. 20, S191 (2005).

${ }^{2}$ M. A. Belkin, F. Capasso, A. Belyanin, D. L. Sivco, A. Y. Cho, D. C. Oakley, C. J. Vineis, and G. W. Turner, Nat. Photonics 1, 288 (2007).

${ }^{3}$ O. V. Kibis, G. Y. Slepyan, S. A. Maksimenko, and A. Hoffmann, Phys. Rev. Lett. 102, 023601 (2009).

${ }^{4}$ D. H. Auston and P. R. Smith, Appl. Phys. Lett. 43, 631 (1983).

${ }^{5}$ Ch. Fattinger and D. Grischkowsky, Appl. Phys. Lett. 54, 490 (1989).

${ }^{6}$ D. H. Auston, K. P. Cheung, J. A. Valdmanis, and D. A. Kleinman, Phys. Rev. Lett. 53, 1555 (1984).

${ }^{7}$ H. G. Roskos, M. D. Thomson, M. Kreß, and T. Löffler, Laser Photonics Rev. 1, 349 (2007).

${ }^{8}$ N. Karpowicz, X. Lu, and X.-C. Zhang, J. Mod. Opt. 56, 1137 (2009).

${ }^{9}$ J. Faist, F. Capasso, D. L. Sivco, C. Sirtori, A. L. Hutchinson, and A. Y. Cho, Science 264, 553 (1994).

${ }^{10}$ R. Köhler, A. Tredicucci, F. Beltram, H. E. Beere, E. H. Linfield, A. G. Davies, D. A. Ritchie, R. C. Iotti, and F. Rossi, Nature 417, 156 (2002).

${ }^{11}$ M. S. Vitiello, G. Scamarcio, V. Spagnolo, T. Losco, R. P. Green, A. Tredicucci, H. E. Beere, and D. A. Ritchie, Appl. Phys. Lett. 88, 241109 (2006).

${ }^{12}$ B. S. Williams, Nat. Photonics 1, 517 (2007).

${ }^{13}$ G. Scalari, C. Walther, J. Faist, H. Beere, and D. Ritchie, Appl. Phys. Lett. 88, 141102 (2006).

${ }^{14}$ P. Y. Han, G. C. Cho, and X.-C. Zhang, Opt. Lett. 25, 242 (2000).

${ }^{15}$ D. D. Arnone, C. M. Ciesla, A. Corchia, S. Egusa, M. Pepper, J. M. Chamberlain, C. Bezant, and E. H. Linfield, Proc. SPIE 3828, 209 (1999).

${ }^{16}$ G. P. Williams, Rep. Prog. Phys. 69, 301 (2006).

${ }^{17}$ K. Reimann, Rep. Prog. Phys. 70, 1597 (2007).

${ }^{18}$ D. Wasserman, C. Gmachl, S. A. Lyon, and E. A. Shaner, Appl. Phys. Lett. 88, 191118 (2006).

${ }^{19}$ V. Ryzhii, Semicond. Sci. Technol. 11, 759 (1996).

${ }^{20}$ H. G. Roskos, M. C. Nuss, J. Shah, K. Leo, D. A. B. Miller, A. M. Fox, S. Schmitt-Rink, and K. Köhler, Phys. Rev. Lett. 68, 2216 (1992).

${ }^{21}$ M. C. Nuss, P. C. M. Planken, I. Brener, H. G. Roskos, M. S. C. Luo, and S. L. Chuang, Appl. Phys. B: Lasers Opt. 58, 249 (1994).

${ }^{22}$ P. C. M. Planken, M. C. Nuss, I. Brener, K. W. Goossen, M. S. C. Luo, S. L. Chuang, and L. Pfeiffer, Phys. Rev. Lett. 69, 3800 (1992).

${ }^{23}$ Y. J. Ding, Q. Hu, M. Kock, and C. E. Stutz, IEEE J. Sel. Top. Quantum Electron. 14, 257 (2008).

${ }^{24}$ N. Sarukura, H. Ohtake, S. Izumida, and Z. Liu, J. Appl. Phys. 84, 654 (1998).

${ }^{25}$ P. Gu and M. Tani, Top. Appl. Phys. 97, 63 (2005).

${ }^{26}$ M. Reid and R. Fedosejevs, Appl. Opt. 44, 149 (2005).

${ }^{27}$ A. M. Weiner, J. Opt. Soc. Am. B 11, 2480 (1994).

${ }^{28}$ H. Xu, E. J. Bjerneld, M. Käll, and L. Börjesson, Phys. Rev. Lett. 83, 4357 (1999).

${ }^{29}$ C. E. Talley, J. B. Jackson, C. Oubre, N. K. Grady, C. W. Hollars, S. M. Lane, T. R. Huser, P. Nordlander, and N. J. Halas, Nano Lett. 5, 1569 (2005).

${ }^{30}$ T. H. Taminiau, F. D. Stefani, F. B. Segerink, and N. F. van Hulst, Nat. Photonics 2, 234 (2008).

${ }^{31}$ S. Kim, J. Jin, Y.-J. Kim, I.-Y. Park, Y. Kim, and S.-W. Kim, Nature 453, 757 (2008).

${ }^{32}$ W. Zhang, A. O. Govorov, and G. W. Bryant, Phys. Rev. Lett. 97, 146804 (2006).

${ }^{33}$ R. D. Artuso and G. W. Bryant, Nano Lett. 8, 2106 (2008).

${ }^{34}$ R. D. Artuso and G. W. Bryant, Phys. Rev. B 82, 195419 (2010).

${ }^{35}$ J. N. Farahani, D. W. Pohl, H. J. Eisler, and B. Hecht, Phys. Rev. Lett. 95, 017402 (2005).

${ }^{36}$ K. T. Shimizu, W. K. Woo, B. R. Fisher, H. J. Eisler, and M. G. Bawendi, Phys. Rev. Lett. 89, 117401 (2002).

${ }^{37}$ A. O. Govorov, G. W. Bryant, W. Zhang, T. Skeini, J. Lee, N. A. Kotov, J. M. Slocik, and R. R. Naik, Nano Lett. 6, 984 (2006).

${ }^{38}$ E. Dulkeith, M. Ringler, T. A. Klar, J. Feldmann, A. Monoz, and W. J. Parak, Nano Lett. 5, 585 (2005).

${ }^{39}$ S. M. Sadeghi, Nanotechnology 20, 225401 (2009).

${ }^{40}$ A. V. Malyshev and V. A. Malyshev, Phys. Rev. B 84, 035314 (2011).

${ }^{41}$ E. Chang, A. S. Sørensen, P. R. Hemmer, and M. D. Lukin, Phys. Rev. Lett. 97, 053002 (2006).

${ }^{42}$ E. Chang, A. S. Sørensen, P. R. Hemmer, and M. D. Lukin, Phys. Rev. B 76, 035420 (2007).

${ }^{43}$ E. Chang, A. S. Sørensen, E. A. Demler, and M. D. Lukin, Nat. Phys. 3, 807 (2007).
${ }^{44}$ F. Oulton, V. J. Sorger, T. Zentgraf, R. M. Ma, L. Dai, G. Bartal, and X. Zhang, Nature 461, 629 (2009).

${ }^{45}$ L. Novotny and B. Hecht, Principles of Nano-Optics (Cambridge University Press, New York, 2006).

${ }^{46}$ A. J. Pasquale, B. M. Reinhard, and L. D. Negro, ACS Nano 5, 6578 (2011).

${ }^{47}$ J. Zhao, B. Frank, S. Burger, and H. Giessen, ACS Nano 5, 9009 (2011).

${ }^{48}$ A. Manjavacas, F. J. Garcia de Abajo, and P. Nordlander, Nano Lett. 11, 2318 (2011).

${ }^{49}$ A. Manjavacas, P. Nordlander, and F. J. Garcia de Abajo, ACS Nano 6, 1724 (2012).

${ }^{50}$ F. H. L. Koppens, D. E. Chang, and F. J. Garcia de Abajo, Nano Lett. 11, 3370 (2011).

${ }^{51}$ S. Nie and S. R. Emory, Science 275, 1102 (1997).

${ }^{52}$ M. Ishifuji, M. Mitsuishi, and T. Miyashita, Appl. Phys. Lett. 89, 011903 (2006).

${ }^{53}$ H. A. Clark, P. J. Campagnola, J. P. Wuskell, A. Lewis, and L. M. Loew, J. Am. Chem. Soc. 122, 10234 (2000)

${ }^{54}$ J.-Y. Yan, W. Zhang, S. Duan, and X.-G. Zhao, J. App. Phys. 103, 104314 (2008).

${ }^{55}$ M. R. Singh, Nanotechnology 24, 125701 (2013).

${ }^{56}$ P. M. Jais, C. von Bilderling, and A. V. Bragas, Papers Phys. 3, 030002 (2011).

${ }^{57} \mathrm{Y}$. Pu, R. Grange, C.-L. Hsieh, and D. Psaltis, Phys. Rev. Lett. 104, 207402 (2010).

${ }^{58}$ U. Hohenester, F. Troiani, E. Molinari, G. Panzarini, and C. Macchiavello, Appl. Phys. Lett. 77, 1864 (2000).

${ }^{59}$ J. Elzerman, K. Weiss, J. M. Sanchez, and A. Imamoglu, preprint arXiv:1011.5203 (2010).

${ }^{60}$ G. Ortner, M. Bayer, Y. Lyanda-Geller, T. L. Reinecke, A. Kress, J. P. Reithmaier, and A. Forchel, Phys. Rev. Lett. 94, 157401 (2005).

${ }^{61}$ Q. Q. Wang, A. Muller, M. T. Cheng, H. J. Zhou, P. Bianucci, and C. K. Shih, Phys. Rev. Lett. 95, 187404 (2005).

${ }^{62}$ A. Govorov, Phys. Rev. B 82, 155322 (2010).

${ }^{63}$ V. V. Klimov, M. Ducloy, and V. S. Letokhov, Eur. Phys. J. D 20, 133 (2002).

${ }^{64}$ J. Gersten and A. Nitzan, J. Chem. Phys. 75, 1139 (1981).

${ }^{65}$ L. Novotny, Appl. Phys. Lett. 69, 3806 (1996).

${ }^{66}$ F. J. García de Abajo and J. Aizpurúa, Phys. Rev. B 56, 15873 (1997).

${ }^{67}$ R. Carminati, J. J. Greffet, C. Henkel, and J. M. Vigoureux, Opt. Commun. 261, 368 (2006)

${ }^{68}$ A. O. Govorov, J. Lee, and N. A. Kotov, Phys. Rev. B 76, 125308 (2007).

${ }^{69}$ Y. V. Vladimirova, V. V. Klimov, V. M. Pastukhov, and V. N. Zadkov, Phys. Rev. A 85, 053408 (2012).

${ }^{70}$ P. B. Johnson and R. W. Christy, Phys. Rev. B 6, 4370 (1972).

${ }^{71}$ K. Bergmann, H. Theuer, and B. W. Shore, Rev. Mod. Phys. 70, 1003 (1998).

${ }^{72}$ M. Weitz, B. C. Young, and S. Chu, Phys. Rev. A 50, 2438 (1994).

${ }^{73}$ A. S. Parkins, P. Marte, P. Zoller, O. Carnal, and H. J. Kimble, Phys. Rev. A 51, 1578 (1995).

${ }^{74}$ T. Pellizzari, S. A. Gardiner, J. I. Cirac, and P. Zoller, Phys. Rev. Lett. 75, 3788 (1995)

${ }^{75}$ C. Monroe, Nature 416, 238 (2002).

${ }^{76}$ P. Kral and M. Shapiro, Rev. Mod. Phys. 79, 53 (2007).

${ }^{77}$ N. V. Vitanov, T. Halfmann, and B. W. Shore, Annu. Rev. Phys. Chem. 52, 763 (2001).

${ }^{78}$ N. V. Vitanov, M. Fleischhauer, B. W. Shore, and K. Bergmann, Adv. At. Mol. Opt. Phys. 46, 55 (2001)

${ }^{79}$ I. R. Sola and V. S. Malinovsky, Phys. Rev. A 68, 013412 (2003).

${ }^{80}$ A. A. Rangelov and N. V. Vitanov, Phys. Rev. A 85, 043407 (2012).

${ }^{81}$ S. T. Cundiff, J. Phys. D 35, R43 (2002).

${ }^{82}$ A. Wirth, M. Th. Hassan, I. Grguraš, J. Gagnon, A. Moulet, T. T. Luu, S. Pabst, R. Santra, Z. A. Alahmed, A. M. Azzeer, V. S. Yakovlev, V. Pervak, F. Krausz, and E. Goulielmakis, Science 334, 195 (2011).

${ }^{83}$ A. Urbańczyk, G. J. Hamhuis, and R. Nötzel, Appl. Phys. Lett. 96, 113101 (2010).

${ }^{84}$ M. Pfeiffer, K. Lindfors, C. Wolpert, P. Atkinson, M. Benyoucef, A. Rastelli, O. G. Schmidt, H. Giessen, and M. Lippitz, Nano Lett. 10, 4555 (2010).

${ }^{85}$ M. Pfeiffer, K. Lindfors, P. Atkinson, A. Rastelli, O. G. Schmidt, H. Giessen, and M. Lippitz, Phys. Status Solidi B 249, 678 (2012). 